\title{
Two-year outcome after recurrent implantation failure: prognostic factors and additional interventions
}

\author{
Christiane Kling • Andreas Schmutzler • Georg Wilke • \\ Jürgen Hedderich • Dieter Kabelitz
}

Received: 14 October 2007 / Accepted: 10 December 2007 / Published online: 12 January 2008

(C) Springer-Verlag 2007

\begin{abstract}
Objectives After recurrent implantation failure (RIF), empirical figures on further prospects are essential for counselling but difficult to estimate within single IVF centres due to high drop-out rates. Alternatively, couples referred to a tertiary unit for RIF were evaluated.

Materials and methods Multi-centre 2-year observational trial of 1,174 eligible couples treated consecutively with adjuvant lymphocyte immunotherapy (LIT) in a university immunological department from 1999 to 2002 after three or more unsuccessful fresh embryo transfers. Acquisition of data was completed in 2005.

Results With another 1.5 oocyte retrievals, delivery rate per couple depended on age (39.3\% at $<30$ years, $16.9 \%$ at $>39$ years, $P<0.005)$. Prognosis was favourable when frozen embryo transfers had been conducted before (34.4 vs. $25.8 \%, P<0.005)$. The outcome was slightly better in ICSI couples as compared to conventional IVF ( 31.0 vs. $24.8 \%$,
\end{abstract}

C. Kling $(\bowtie) \cdot$ D. Kabelitz

Institute of Immunology,

University Medical Center Schleswig-Holstein,

Michaelisstr. 5, 24105 Kiel, Germany

e-mail: kling@immunologie.uni-kiel.de

\section{A. Schmutzler}

Department of Obstetrics and Gynaecology,

IVF Unit, University Medical Center Schleswig-Holstein,

Kiel, Germany

G. Wilke

Fertility Center, Hildesheim, Germany

\section{J. Hedderich}

Department of Medical Informatics and Statistics,

University Medical Center Schleswig-Holstein,

Kiel, Germany
$P<0.05)$. Birth rates per fresh embryo transfer from the fourth to eighth retrieval were 17.4-18.3-15.0-12.9-12.9\% (decline not significant). Apart from LIT, further additional interventions were given more often to couples who had had frozen embryo transfers before (49 vs. $40 \%$, $P<0.005$ ).

Conclusions Female age and ovarian response are crucial for further IVF prognosis. Previous frozen embryo transfers indicate better chances. Couples with male factor infertility may benefit from intracytoplasmatic sperm injection (ICSI) because underlying female factors are less prevalent. Cycle rank had comparatively little impact. Additional interventions are preferentially offered to couples who have a favourable prognosis anyway. Their multiple use is common practice in RIF, but its value should be considered limited.

Keywords Delivery rate - German embryo protection law . Embryo cryopreservation - Intracytoplasmatic sperm injection (ICSI) · Immunotherapy

\section{Introduction}

Recurrent implantation failure (RIF) is a distressing situation in the in vitro fertilization (IVF) programme which is difficult to relieve. Realistic figures on the long-term prognosis are necessary for counselling but difficult to establish in single IVF centres due to high drop-out rates. After the third IVF treatment cycle, drop-out rates between 22 and $50 \%$ are reported with a tendency to increase with each consecutive cycle [14, 17, 18, 24]. Statistical extrapolation is recognised to create figures which may overestimate success rates [2]. National IVF registries are based on evaluation of treatment cycles, 
but as a disadvantage they are not centred on patient courses.

In this study, RIF couples treated consecutively at the Institute of Immunology at the Kiel University Hospital between 1999 and 2002, were followed up for another 2 years. The outcome of pregnancies was registered until summer 2005. The patients had been referred for supportive lymphocyte immunotherapy (LIT) after three or more unsuccessful fresh embryo transfers from 47 national IVF centres, that is $40 \%$ of the 113 centres registered in Germany in 2002. Cross-sectional empirical data on ongoing IVF therapy were obtained prospectively and analysed in order to illustrate the influence of prognostic factors derived from the past IVF history.

\section{Materials and methods}

Lymphocyte immunotherapy (LIT)

At the Kiel Institute of Immunology, the treatment is introduced for patients with RIF and recurrent miscarriages after exclusion diagnosis [7] and is covered by the national health insurance carriers. A small proportion (approximately 5\%) referred for immunological treatment were precluded for other non-gynaecological causes, e.g. infectious or malignant disease of the male partner, HLA identical partners, female diabetes, systemic lupus erythematosus, or antiphospholipid syndrome, as described in detail elsewhere $[9,10]$.

\section{Questionnaires}

From 1999 to 2002, 1,700 women underwent LIT after three or more fresh IVF cycles and consented to be interviewed 2 years later. In October 2002, all couples treated from 1999 to 2000, and in October 2003 those treated in 2001 and 2002 received a questionnaire. The referring gynaecologists got questionnaires on the IVF outcome 3 months later. The couples were referred to us by 47 of 113 IVF centers registered in Germany in 2002. In 36 cases, the couples were referred by their general gynaecologists.

The couples and practitioners were interviewed on the course of infertility treatment with the same partner, deliveries beyond the 23rd gestational week, and adjuvant therapies apart from LIT. We received informative feedback from 1,435 of 1,700 couples ( $84.4 \%$ ), that is 1,078 couples $(63.4 \%)$ by questionnaire (935) and/or telephone (143). Another 357 cases $(21.0 \%)$ were reported by the gynaecologists (and for 287 of them, we had double responses). The concept of the questionnaire was approved by the university's data protection registrar; approval of the ethics committee was not required.
IVF history and definition of RIF

The term RIF referred to couples who had at least three fresh embryo transfers in the past, and the reference date for female age at all further treatments was the first LIT. IVF (in vitro fertilisation) had been initiated for tubal, mild andrologic, and idiopathic causes of infertility, endometriosis, polycystic ovary syndrome, and anovulation. ICSI (intracytoplasmatic sperm injection) had been started in markedly reduced sperm counts, reduced fertilisation rate, low responders, or lack of fertilisation in an IVF attempt. Those oocyte retrievals which led to transfers of fresh and cryopreserved embryos were considered for evaluation. Additional frozen embryo transfers were included into the calculation of birth rates per oocyte retrieval. Since there were no reports of severe overhydration-hyperstimulation syndrome (OHSS) which prevented fresh transfer, the number of evaluated oocyte retrievals equal the number of fresh embryo transfers. The evaluation ended at the first delivery after embryo transfer or with the status of the questionnaire if the couple did not conceive.

According to the guidelines of the German Medical Association published in 1998, two embryos were recommended for cultivation for women up to 35 years and three embryos for elder women.

According to the German IVF registry, in the years 2000-2004, 83-85\% of all started stimulation cycles and $88-90 \%$ of oocyte retrievals led to embryo transfer. From 2001 on, the short stimulation protocol with GnRH agonists was used in $12-13 \%$, the long protocol in $62-65 \%$, antagonists in $20-25 \%$, none in $1-2 \%$ of all treatments, recombinant FSH being the prevalent gonadotropin in Germany (63\% of stimulation cycles).

German embryo protection law and health insurance modalities

A maximum of three embryos can be selected for cultivation at the pronucleus stage for transfer after up to 5 days. Additional pronuclei can be cryopreserved. Embryo selection beyond the pronucleus stage, surrogacy, and oocyte donation are prohibited. Up to four IVF/ICSI cycles were paid for by the health insurance companies until the end of 2003.

\section{Statistics}

Means, standard deviation, odds ratio, and range are given for female age, delivery rate, embryo transfers, and oocyte retrievals in descriptive intention. $\chi^{2}$ test was also applied for differences of the characteristics of patient subgroups. The Cochran trend test was used for delivery rates related 
to female age. For all statistics a value of $P<0.05$ was considered significant.

\section{Results}

Characteristics of RIF couples

Of 1700 couples, 265 were lost to follow-up and 1,435 were informative. One hundred and eighty-three of these reported that they did not have any more transfers. In the group of 265 couples lost to follow-up, $46 \%$ had had at least four oocyte retrievals, and the loss of follow-up rate increased with female age. Thus financial constraints and individual factors related to advanced age may have led to discontinuation of IVF therapy. As judged by the women's name, $23 \%$ in this group (62/265 patients) probably had linguistic problems and may have preferred to continue IVF in their home country [8]. In contrast, this applied for $11 \%$ in the informative group $(163 / 1435$ patients, $p<0.001)$.

After LIT, 1,252 couples continued IVF therapy in German centres. Of these, 78 had transfers of frozen-thawed embryos derived from the previous cycles only, and were excluded from evaluation.

Thus 1,174 couples were eligible. Mean female age at LIT was $34.2 \pm 2.9$ years (range $21-43$ ), duration of infertility $5.8 \pm 2.3$ years (range $1-20$ ). On average, prior to LIT $3.7 \pm 0.8$ fresh cycles (3-21) and $4.2 \pm 1.2$ embryo transfers (fresh and frozen, 3-21) had been conducted. The majority (701 couples, 60\%) had three oocyte retrievals, 306 couples $(26 \%)$ had four retrievals, 92 (8\%) five retrievals, $42(3 \%)$ six retrievals, and $33(3 \%)$ had 7-21 retrievals. Prior and post LIT, on average 2.5 embryos per transfer were given. Male factor infertility was an underlying cause in 728 couples, and in 473 (40\%), it was the only factor. Tubal occlusion was found in 371 patients, and in $171(15 \%)$ it was the single cause. Endometriosis was reported in 135 women, other causes in 231 women, contributing to mixed etiologies in 398 couples (34\%). Thirty-three couples $(3 \%)$ were defined to have idiopathic infertility. Ninty-four cases (8\%) were unspecified. Of 1,174 couples, $531(45 \%)$ were treated by ICSI from the beginning. In 68 of them, ICSI had been performed with sperm obtained from testicular extraction (TESE) or microepididymal aspiration (MESA). Three hundred and seventy-one couples (32\%) had conventional IVF only. The remaining 272 couples (23\%) had IVF as well as ICSI cycles.

Within 2 years after LIT, 1174 IVF couples had another $1.5 \pm 0.7(1-8)$ retrievals and $1.7 \pm 0.8(1-10)$ transfers. In summary, the whole IVF treatment comprised $5.2 \pm 1.1(4-$ 23) oocyte retrievals and $6.0 \pm 1.5$ (4-23) transfers per couple. The highest overall amount of oocyte retrievals leading to birth was 14 , and the highest number of transfers was 17 .
Prognostic factors after recurrent implantation failure

\section{Cycle rank}

Within 2 years, birth rates per fresh embryo transfers and per couples showed a tendency to decline after the third to the seventh unsuccessful oocyte retrieval. This trend was not statistically significant (Fig. 1). When frozen embryo transfers had been possible during the preceding cycles, the delivery rate per oocyte retrieval stayed as high as $20 \%$ (data not shown).

\section{Female age}

After 1.5 additional fresh transfers, 333 of 1,174 women delivered $(28.4 \%$, Table 1$)$. The average birth rate per fresh embryo transfer was $17.3 \%$, per frozen transfer $8.2 \%$, and $18.6 \%$ per oocyte retrieval cumulating fresh and frozen transfers. As expected, delivery rates were highly dependent on female age (Fig. 2). Women below 30 years reached a 2-year delivery rate of $39 \%$, from 30 to 39 years it was $30-25 \%$, and at age 40 years and older $17 \%$. The eldest mother was 42 years old at LIT.

\section{Embryo cryopreservation}

Overall 310 of 333 deliveries (93\%) originated from fresh IVF cycles (conventional IVF 102, ICSI 208), and 23 (7\%) from transfer of frozen-thawed embryos. The prognosis was favourable when frozen embryo transfers had been possible in the past, as compared to couples without cryopreservation cycles (Table 1, Fig. 3). After LIT, both groups used embryo cryopreservation, but the proportion of frozen embryo transfers was twice as high in those who had had cryopreservation before LIT (18.7 vs. 9.9\%, $p<<0.0001)$. This was combined with a better outcome per oocyte retrieval as well as per fresh embryo transfer. When the number of previous frozen embryo transfers was taken as a

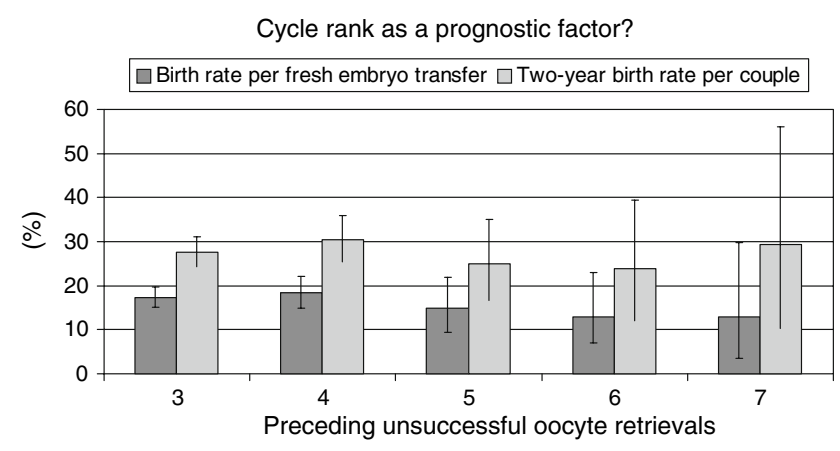

Fig. 1 Number of couples with three to seven unsuccessful retrievals: $701-306-42-17$, female age $33.7 \pm 2.9, \quad 34.5 \pm 2.9,35.1 \pm 3.0$, $35.7 \pm 2.6,35.8 \pm 2.6$ years. Decline in birth rates not significant (Cochran trend test) 


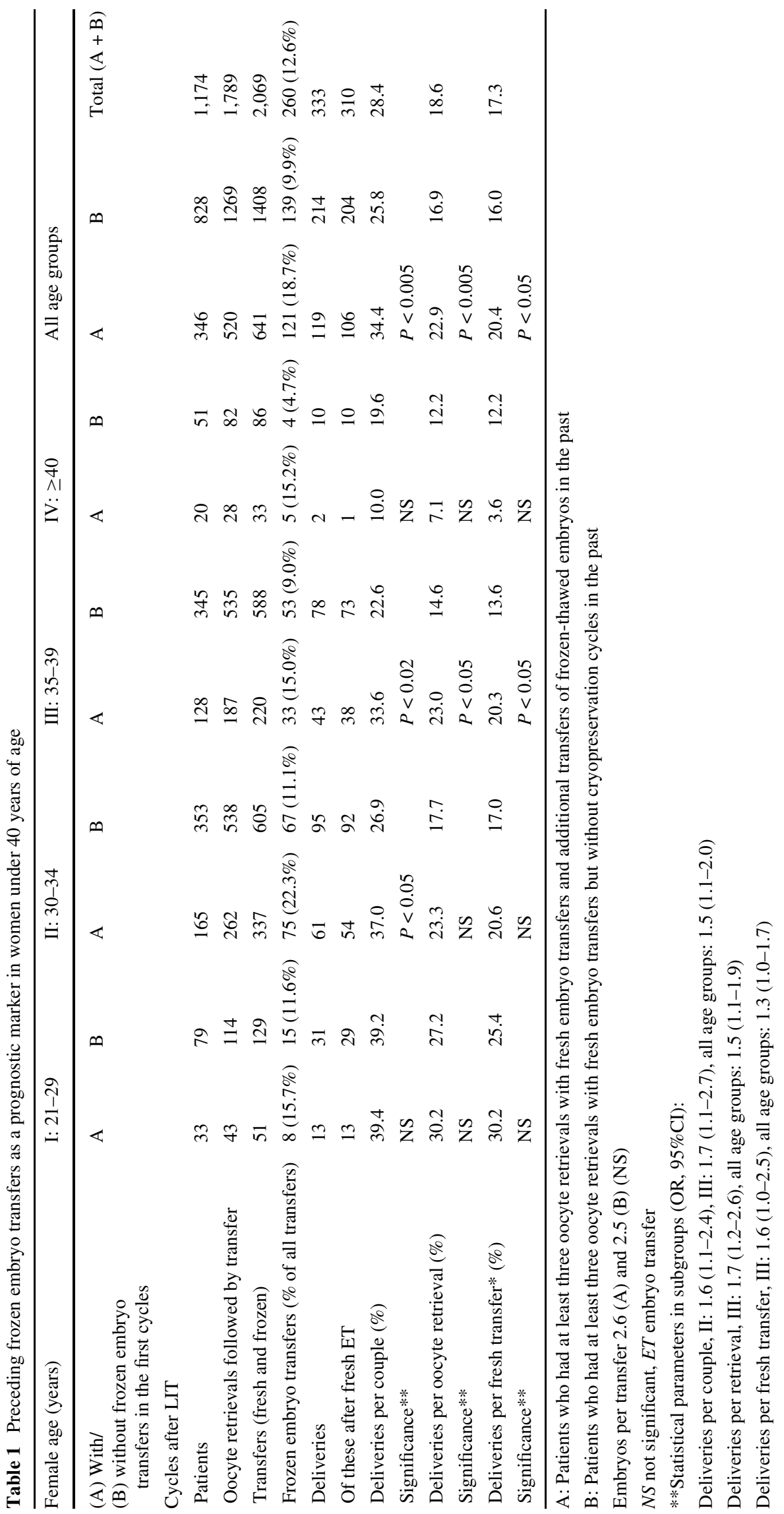


Female age as a prognostic marker in further IVF and ICSI treatment

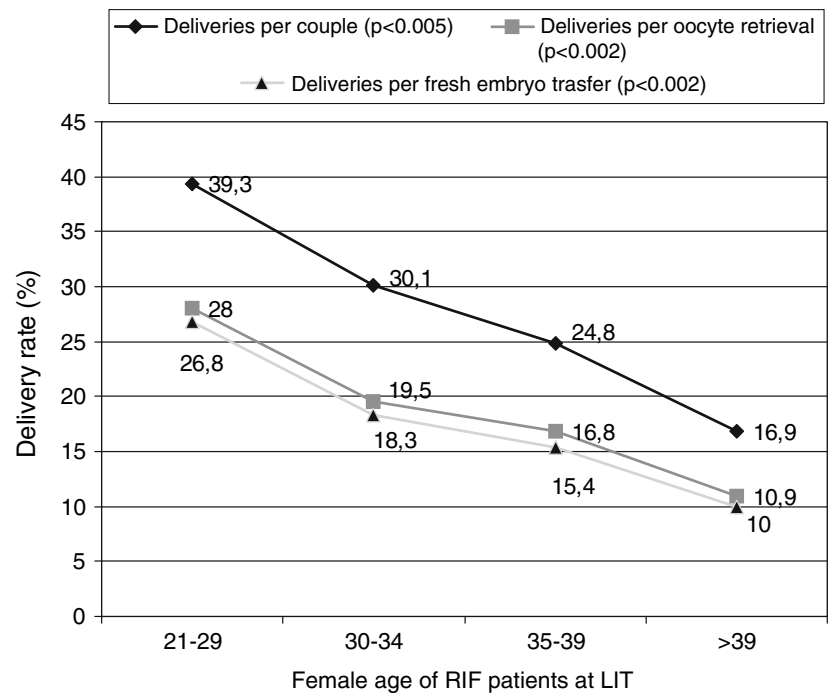

Fig. 2 Delivery rates per oocyte retrievals include fresh and frozen embryo transfers. Number of women below 30 years: $n=112,30$ 34 years: $n=537,35-39$ years: $n=473$, over 39 years: $n=71$. Decline of delivery rates with advancing female age is statistically significant (Cochran trend test)

Frozen embryo transfers in the first cycles as a prognostic marker in women under 40 years of age

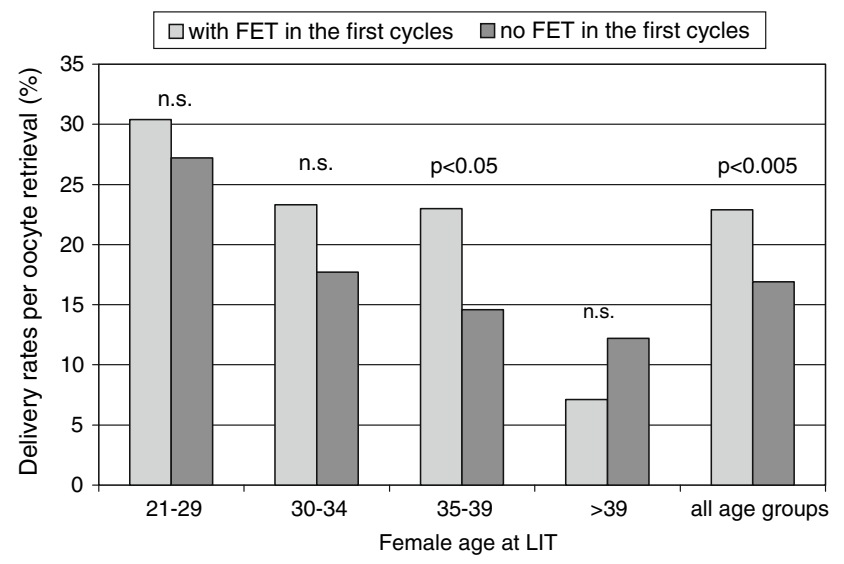

Fig. 3 Delivery rates per oocyte retrieval achieved after LIT are higher when cryopreservation had been possible in the first cycles. The figure illustrates the results given in Table $1, N S$ not significant

marker for ovarian response, the correlation was also significantly positive (Fig. 4).

\section{Male factor infertility}

When the mode of fertilisation over the whole course of IVF treatment is evaluated, RIF couples treated with ICSI from the beginning had a better outcome than those treated with conventional IVF (Table 2). Of those who had had cycles with as well as without ICSI, most switched from conventional IVF to ICSI at some stage of the treatment. They achieved intermediate birth rates. Characteristics like
Frozen embryo transfers in the first cycles as a prognostic marker in RIF

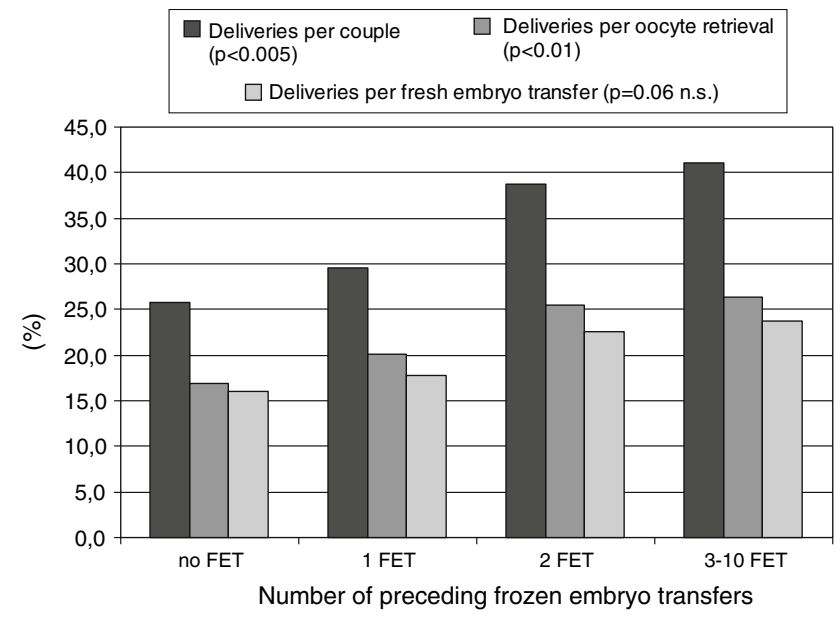

Fig. 4 Frozen embryo transfers (FET) in the first cycles suggest a good oocyte/ embryonic quality in further IVF treatment. There is a positive correlation between delivery rates and number of FET (Cochran trend test). Couples without FET: $n=828$, 1 FET: $n=183$, 2 FET: $n=90,3-10$ FET: $n=73$. Mean female age at LIT and mean number of oocyte retrievals before LIT did not differ significantly between the groups $(34.2 \pm 3.0,34.4 \pm 2.8,33.5 \pm 2.8,33.9 \pm 2.9$ years $/ 3.7 \pm$ $0.8,3.7 \pm 0.8,3.7 \pm 0.8,3.9 \pm 0.9$ retrievals, respectively)

female age and rate of cryopreservation were similar in all three groups.

\section{Additional interventions}

Various adjuvant measures which are thought to improve implantation by influencing immune function, endometrial haemostaseology, or embryonic capacities to implant were combined and given at various stages of IVF therapy additionally to LIT. Examples are corticosteroids, immunoglobulins, LeukoNorm CytoChemia ${ }^{\circledR}$, Sildenafil (vaginally), Pentoxifylline, and polar body biopsy. Most often aspirin, low dose heparin, and laser-assisted hatching were mentioned. In some cases low dose heparin was prescribed for thromboprophylaxis in ovarian hyperstimulation syndrome (OHSS) or hereditary coagulation defects. In the cycles following LIT, 499 patients (43\%) were treated additionally, while 275 (23\%) were not. In 401 (34\%) no information was given so that the number of unreported cases may be high.

Couples who had had cryopreservation before received additional interventions significantly more often than couples who did not have cryopreservation: 49 versus $40 \%$ (170/346 couples vs. $328 / 828$ couples, $P<0.005$ ).

\section{Discussion}

Our evaluation of a large RIF group treated in Germany showed a mean delivery rate of $18.6 \%$ per successful oocyte 
Table 2 Two-year outcome after conventional IVF or ICSI ( 1,174 couples $)$

The characteristics are similar in all subgroups

* Significant differences are only noted for birth rates per couple $(P<0.05)$ and per oocyte retrieval $(P<0.05)$ between the ICSI and conventional IVF subgroups

\begin{tabular}{llll}
\hline $\begin{array}{l}\text { Mode of fertilisation } \\
\text { from the first cycle on }\end{array}$ & ICSI & $\begin{array}{l}\text { Conventional } \\
\text { IVF }\end{array}$ & $\begin{array}{l}\text { IVF and } \\
\text { ICSI cycles }\end{array}$ \\
\hline $\begin{array}{l}\text { Couples } \\
\text { Characteristics }\end{array}$ & 531 & 371 & 272 \\
$\begin{array}{l}\text { Female age at LIT (years) } \\
\text { Fresh embryo transfers before }\end{array}$ & $33.9 \pm 2.8$ & $34.3 \pm 3.1$ & $34.6 \pm 3.0$ \\
$\quad$ LIT (per couple) & $1,928(3.6)$ & $1,346(3.6)$ & $1050(3.9)$ \\
Fresh embryo transfers after & $802(1.5)$ & $566(1.5)$ & $421(1.5)$ \\
$\quad$ LIT (mean per couple) & $121(13.1 \%)$ & $76(11.8 \%)$ & $63(13.0 \%)$ \\
Frozen embryo transfers & 165 & & 76 \\
$\quad(\%$ of all transfers after LIT) & 153 & 92 & 71 \\
Deliveries & 12 & 86 & 5 \\
After fresh embryo transfer & & 6 & 27.9 \\
After frozen embryo transfer & $31.0^{*}$ & & 18.1 \\
Birth rates & $20.6^{*}$ & $24.8^{*}$ & 16.9 \\
Per couple (\%) & 19.1 & $16.3 *$ & 15.2 \\
Per oocyte retrieval $(\%)$ & & & \\
Per fresh embryo transfer $(\%)$ & & & \\
\hline
\end{tabular}

retrieval where frozen embryo transfers are included, $17.3 \%$ per fresh embryo transfer, and $8.2 \%$ per frozen embryo transfer. Since $84 \%$ of stimulation cycles lead to embryo transfer according to the German IVF registry, the delivery rate per started cycle is estimated to be $15.6 \%$.

Bias caused by preselection of the practitioners referring to our institute and by LIT (see "Materials and methods") cannot be totally excluded. Since patients were accepted irrespective of the embryonic quality in their previous cycles, and exclusion criteria for LIT were non-gynaecological and applied rarely (see "Materials and methods"), we assume that the bias was small. According to the data evaluated by the German IVF registry, the overall birth rates per fresh embryo transfer in 2001 were $18.4 \%$ after IVF cycles, $18.8 \%$ after ICSI cycles, and $10.2 \%$ after frozen embryo transfer [1]. Thus the results presented here appear to be plausible.

Several studies have shown that the birth rate per embryo transfer is optimal over the first three stimulation cycles and somewhat declines thereafter [13, 18, 20, 24]. The estimations of cumulative birth rates suggest that the chances to conceive are reduced but stable up to the sixth to eighth cycle [25]. In our cohort, differentiation for cycle rank did not reveal a significant correlation with the number of preceding unsuccessful retrievals, either (Fig. 1). Although the German legislation is unique for prohibiting embryo selection after the pronucleus stage, the outcomes per cycle in our cohort are in line with international figures available for RIF (Table 3).

Female age and embryo cryopreservation

Female age and the response to ovarian stimulation are significant prognostic factors of further outcome. An indicator of the ovarian reserve is the number of well developed oocytes per stimulation cycle [18] and of the embryos gained consecutively [4]. Here additional frozen embryo transfers were taken as a marker of ovarian reserve. If frozen embryo transfers had been possible in the first cycles, women in their thirties can be regarded younger biologically. They did not only retain a better ovarian response and thus gained more transfers, but also had a significantly higher chance to conceive per fresh embryo transfer.

Frozen embryo transfers are less successful, and the procedure of cryopreservation and storage produces extra costs for the patients. Therefore the method was not uniformly encouraged by the referring IVF centres. If the policy had been standardized in favour of cryopreservation, the difference between the groups who had or did not have frozen embryo transfers in their first cycles would possibly have been even more accentuated than in the present evaluation.

Selection of cultivated embryos by morphological criteria or preimplantation genetic diagnosis (PGD) is seen as an approach to increase the success rates per embryo transfer. However, it cannot be offered to couples who have no embryos in excess although this group may be at a higher risk for embryonic disturbances. On the other hand, women with excess embryos may benefit from their generally higher embryonic quality and not only from embryo selection [3].

\section{Other factors}

Where ICSI is applied for male infertility as in our cohort, the basic difference between those who were treated by 


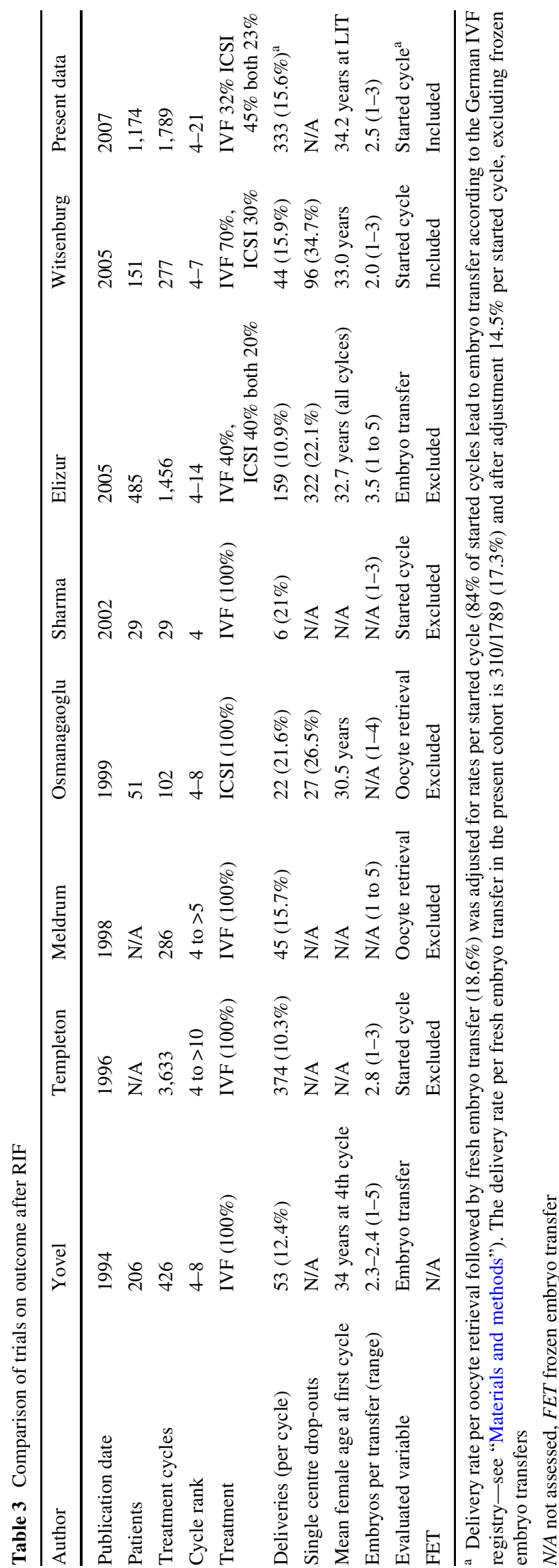

ICSI and those who received conventional IVF are underlying female factors of infertility. Stage III/IV endometriosis [12], sactosalpinges or other tubal disease [15], uterine fibroids [6], and reduced endometrial responsiveness [16] lead to impaired oocyte quality or uterine function. This may be the main reason why the birth rate is somewhat higher in ICSI cycles. When ICSI is applied in non-male infertility, there is no significant difference to conventional IVF [23].

Notably, lifestyle factors like smoking and female obesity have been shown to impair the chances to conceive $[11,26]$ and with some support can actively be modified by the patients themselves.

\section{Additional interventions}

With good ovarian response, the chances in further IVF or ICSI treatment remain favourable, although not only fresh embryo cycles, but also frozen embryo transfers may have failed. This circumstance is difficult to explain to couples with RIF. Therefore additional diagnostic or therapeutic measures are frequently demanded [21, 22]. Two-thirds of the IVF centres in Britain offer various immunological and non-immunological interventions whose efficacy is under discussion [19]. In the USA, at least $78 \%$ of patients with reproductive failure were offered different therapies including aspirin, heparin, and/or steroids [5]. Multiple interventions seem to be common practice in RIF. Nevertheless, our data indicate that they were preferentially offered to couples who had a favourable prognosis anyway. This can lead to the erroneous conclusion that the benefit was due to additional therapies. When planning a trial on additional interventions in RIF, randomisation is necessary for female age and for the ovarian response. IVF and ICSI indications should be differentiated. The least important factor is the number of previous cycles from the fourth on.

\section{Conclusions}

The evaluation confirms that significant prognostic factors are female age, the ovarian response to controlled hyperstimulation, and to a lesser extent, the underlying disorder of infertility. Couples who are treated with ICSI due to male factor infertility have a slightly better prognosis than those who are treated with conventional IVF because underlying female factors are less prevalent. A significant influence of cycle rank was not detectable in our cohort.

Concomitant interventions have been used to support implantation, but the value of their multiple use may be limited. They may be inferior to the option to modify lifestyle habits. 
Acknowledgments In the Institute of Immunology, Julia Magez, Andrea Iwersen, and Kristina Dudda considerably supported data collection. We appreciate the cooperation of our patients and of the referring IVF centres organized in the German Federal Association of Reproductive Medical Centres (BRZ, URZ, list with the authors).

\section{References}

1. Andersen AN, Gianaroli L, Felberbaum R, de Mouzon J, Nygren KG (2005) Assisted reproductive technology in Europe, 2001. Results generated from European registers by ESHRE. Hum Reprod 20:1158-1176

2. Daya S (2005) Life table (survival) analysis to generate cumulative pregnancy rates in assisted reproduction: are we overestimating our success rates? Hum Reprod 20:1135-1143

3. Elizur SE, Lerner-Geva L, Levron J, Shulman A, Bider D, Dor J (2005) Factors predicting IVF treatment outcome: a multivariate analysis of 5310 cycles. Reprod Biomed Online 10:645-649

4. German Society of Obstetrics and Gynecology (2006) (Ärztekammer Schleswig- Holstein) (ed), German IVF Registry-yearbook 2005, Bad Segeberg, pp 1-31

5. Ghazeeri GS, Kutteh WH (2001) Immunological testing and treatment in reproduction: frequency assessment of practice patterns at assisted reproduction clinics in the USA and Australia. Hum Reprod 16:2130-2135

6. Khalaf Y, Ross C, El Toukhy T, Hart R, Seed P, Braude P (2006) The effect of small intramural uterine fibroids on the cumulative outcome of assisted conception. Hum Reprod 21:2640-2644

7. Kling C., Magez J, Jenisch S, Kabelitz D (2002) [Effect of allogeneic leukocyte immunization on consecutive IVF/ICSI treatment for failure in the in-vitro fertilization program]. Geburtsh Frauenheilk 62:661-667

8. Kling C., Wilke G, Schmutzler A, Hedderich J, Kabelitz D (2007) Outcome two years after recurrent IVF implantation failure. Geburtsh Frauenheilk 67:1002-1008

9. Kling C, Steinmann J, Flesch B, Westphal E, Kabelitz D (2006a) Transfusion-related risks of intradermal lymphocyte immunotherapy: Single cases in a large cohort and review of the literature. Am J Reprod Immunol 56:157-171

10. Kling C, Steinmann J, Westphal E, Magez J, Kabelitz D (2006b) Adverse effects of intradermal allogeneic lymphocyte immunotherapy: acute reactions and role of autoimmunity. Hum Reprod 21:429-435

11. Klonoff-Cohen H (2005) Female and male lifestyle habits and IVF: what is known and unknown. Hum Reprod Update 11:179203

12. Kuivasaari P, Hippelainen M, Anttila M, Heinonen S (2005) Effect of endometriosis on IVF/ICSI outcome: stage III/IV endometriosis worsens cumulative pregnancy and live-born rates. Hum Reprod 20:3130-3135
13. Meldrum DR, Silverberg KM, Bustillo M, Stokes L (1998) Success rate with repeated cycles of in vitro fertilization-embryo transfer. Fertil Steril 69:1005-1009

14. Osmanagaoglu K, Tournaye H, Camus M, Vandervorst M, Van Steirteghem A, Devroey P (1999) Cumulative delivery rates after intracytoplasmic sperm injection: 5 year follow-up of 498 patients. Hum Reprod 14:2651-2655

15. Ozmen B, Diedrich K, Al Hasani S (2007) Hydrosalpinx and IVF: assessment of treatments implemented prior to IVF. Reprod Biomed Online 14:235-241

16. Richter KS, Bugge KR, Bromer JG, Levy MJ (2007) Relationship between endometrial thickness and embryo implantation, based on 1,294 cycles of in vitro fertilization with transfer of two blastocyststage embryos. Fertil Steril 87:53-59

17. Schroder AK, Katalinic A, Diedrich K, Ludwig M (2004) Cumulative pregnancy rates and drop-out rates in a German IVF programme: 4102 cycles in 2130 patients. Reprod Biomed Online 8:600-606

18. Sharma V, Allgar V, Rajkhowa M (2002) Factors influencing the cumulative conception rate and discontinuation of in vitro fertilization treatment for infertility. Fertil Steril 78:40-46

19. Tan BK, Vandekerckhove P, Kennedy R, Keay SD (2005) Investigation and current management of recurrent IVF treatment failure in the UK. BJOG 112:773-780

20. Templeton A, Morris JK, Parslow W (1996) Factors that affect outcome of in-vitro fertilisation treatment. Lancet 348:1402-1406

21. Urman B, Yakin K, Balaban B (2005a) Recurrent implantation failure in assisted reproduction: how to counsel and manage. A. General considerations and treatment options that may benefit the couple. Reprod Biomed Online 11:371-381

22. Urman B, Yakin K, Balaban B (2005b) Recurrent implantation failure in assisted reproduction: how to counsel and manage. B. Treatment options that have not been proven to benefit the couple. Reprod Biomed Online 11:382-391

23. van Rumste MM, Evers JL, Farquhar CM (2003) Intra-cytoplasmic sperm injection versus conventional techniques for oocyte insemination during in vitro fertilisation in patients with non-male subfertility. Cochrane Database Syst Rev CD001301

24. Witsenburg C, Dieben S, van der Westerlaken L, Verburg H, Naaktgeboren N (2005) Cumulative live birth rates in cohorts of patients treated with in vitro fertilization or intracytoplasmic sperm injection. Fertil Steril 84:99-107

25. Yovel I, Geva E, Lessing JB, Yaron Y, Botchan A, Amit A (1994) Analysis of the fourth to eighth in-vitro fertilization treatments after three previously failed attempts. Hum Reprod 9:738-741

26. Zitzmann M, Rolf C, Nordhoff V, Schrader G, Rickert-Fohring M, Gassner P, Behre HM, Greb RR, Kiesel L, Nieschlag E (2003) Male smokers have a decreased success rate for in vitro fertilization and intracytoplasmic sperm injection. Fertil Steril 79(Suppl 3):1550-1554 